\title{
Novel needle cutting edge geometry for end-cut biopsy
}

Jason Z. Moore ${ }^{\text {a) }}$

Department of Mechanical and Nuclear Engineering, Pennsylvania State University, State College, Pennsylvania 16802

Patrick W. McLaughlin

Department of Radiation Oncology, University of Michigan, Ann Arbor, Michigan 48109

\begin{abstract}
Albert J. Shih
Department of Mechanical and Nuclear Engineering, Pennsylvania State University, State College, Pennsylvania 16802 and Department of Biomedical Engineering, University of Michigan, Ann Arbor, Michigan 48109
\end{abstract}

(Received 14 February 2011; revised 11 September 2011; accepted for publication 10 November 2011; published 12 December 2011)

Purpose: To introduce and determine the biopsy length performance of the novel enhanced cutting edge (ECE) needle tip design, which contains high inclination angles that allow for more efficient tissue cutting.

Methods: ECE and regular two-plane symmetric needle tip's biopsy performance and cutting force are compared over a series of needle insertion experiments into bovine liver under varying levels of internal needle vacuum. An earlier developed needle tip force model is also applied. From these experiments and force model, the effect of needle tip geometry and vacuum on biopsy performance and force is studied.

Results: Biopsy sample length is on average 22\%, 30\%, and 49\% longer for ECE needles compared to that of regular needles for the internal pressures of $0,-33.9$, and $-67.7 \mathrm{kPa}$, respectively. For ECE needles the vacuum level of $-67.7 \mathrm{kPa}$ produces on average biopsy lengths that are $41 \%$, $31 \%, 29 \%, 45 \%$, and $42 \%$ longer compared to no vacuum for two-plane needle tip bevel angles of $10^{\circ}, 15^{\circ}, 20^{\circ}, 25^{\circ}$, and $30^{\circ}$, respectively. The force results show the ECE needle can be inserted with less initial insertion force than the regular two-plane needle for needles where the needle tip is fully contacting the tissue upon insertion. Vacuum is also showed to help lower insertion forces.

Conclusions: The novel ECE needle tip design outperforms the regular two-plane symmetric needle by yielding longer biopsy samples and lower insertion forces, thereby demonstrating the benefits of needle geometries that contain higher inclination angles. The use of vacuum further improves the ECE needle tip biopsy sample length and lowers insertion forces. (C) 2012 American Association of Physicists in Medicine. [DOI: 10.1118/1.3665253]

Key words: biopsy, tissue cutting, needle geometry

\section{INTRODUCTION}

Needle biopsy is a common medical procedure where a hollow needle is used as a minimally invasive means to extract tissue from the body for diagnosis of cancer and other disease. End-cut needle biopsy is a biopsy procedure, as shown in three steps in Fig. 1, which can acquire a large volume of tissue sample in a minimally invasive procedure. In the first step of end-cut biopsy, the hollow needle and stylet (solid rod) are positioned directly in front of the target area. In the second step, the hollow needle advances forward cutting the tissue and a mechanism seals the sample inside the needle. In the third step, the needle is removed and the biopsy sample is examined. The length of the biopsy sample is usually less than the needle insertion length due to inefficiencies in cutting by the needle tip edge geometry and the resistance due to friction of tissue moving inside the needle.

The ability of a biopsy needle to efficiently cut tissue and acquire a large amount of tissue sample is crucial for a successful diagnosis of diseases that cannot be easily imaged using medical equipment (CT, MRI, etc.) such as prostate cancer. Diseases that can be imaged such as breast cancer are targeted with a needle using imaging equipment and a specific sample of tissue is acquired. However, in the case of prostate biopsy, long cores of tissue are required to sample all areas of the prostate. Prostate biopsy needles that acquire smaller samples of tissue have a higher occurrence of false negative results, ${ }^{1-3}$ which leads to delayed treatment of the patient. This paper explores methods to increase sample length of an end-cut biopsy needle through advanced needle tip cutting edge geometry and the use of vacuum.

Some biopsy devices apply a vacuum pressure inside the hollow needle (negative internal needle pressure) to improve biopsy sample length, a common practice in many breast biopsy devices. It has been shown, in breast biopsy, that a 14 gauge vacuum assisted end-cut biopsy device can obtain more samples faster than with a regular 14 gauge needle device. ${ }^{4}$ However, quantified studies of vacuum level on endcut biopsy performance have not been well documented.

Solid needle insertion force and deflection into tissue have been extensively explored in a number of studies. 
Step 1

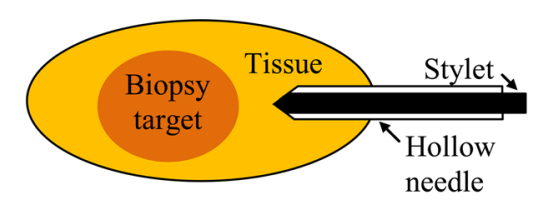

Step 2

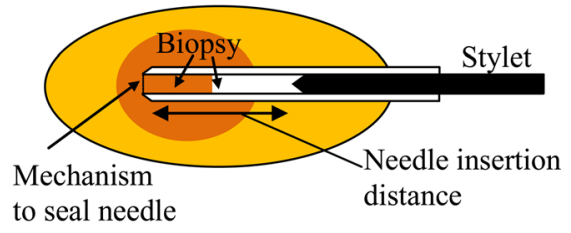

Step 3

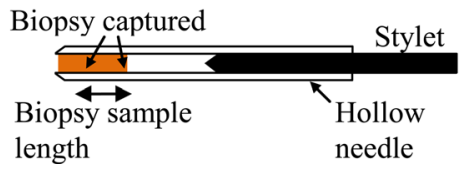

FIG. 1. End-cut needle biopsy procedure of capturing tissue.

Podder et al. ${ }^{5}$ and Maurin et al. ${ }^{6}$ studied the in vivo solid needle force. The effect of tip geometry on solid needle deflection and force has been measured experimentally ${ }^{7,8}$ and modeled. ${ }^{9-11}$ However, because this work is based on solid needle cutting, these studies cannot be directly applied to hollow biopsy needle performance. The survey shows that results are lacking for hollow needle biopsy performance in relation to needle cutting edge geometry and vacuum.

Moore et al. ${ }^{12-15}$ has explored both flat plane and curved plane needle tips to analytically solve the inclination angle $(\lambda)$, rake angle $(\alpha)$, and contact length $(l)$ of the needle tip geometry. The $\lambda$ in cutting mechanics is defined as the angle between the tangent to the cutting edge, vector $\mathbf{s}$, and the plane perpendicular to the cutting direction, plane $\mathbf{P}_{\mathbf{r}}$, as illustrated in Fig. 2. The $\alpha$ is defined as the angle between planes $\mathbf{P}_{\mathbf{r}}$ and $\mathbf{A}_{\gamma}$ measured in plane $\mathbf{P}_{\mathbf{n}}$ as shown in Fig. 2, where $\mathbf{P}_{\mathbf{n}}$ is the plane with normal vector $\mathbf{S}$ and $\mathbf{A}_{\gamma}$ is the face plane of the needle tip surface. In Fig. 2 vector a marks, the intersection of planes $\mathbf{P}_{\mathbf{n}}$ and $\mathbf{A}_{\gamma}$ and vector $\mathbf{b}$ marks the intersection of planes $\mathbf{P}_{\mathbf{n}}$ and $\mathbf{P}_{\mathbf{r}}$; therefore, making the angle between $\mathbf{a}$ and $\mathbf{b}$ equal to $\alpha$.

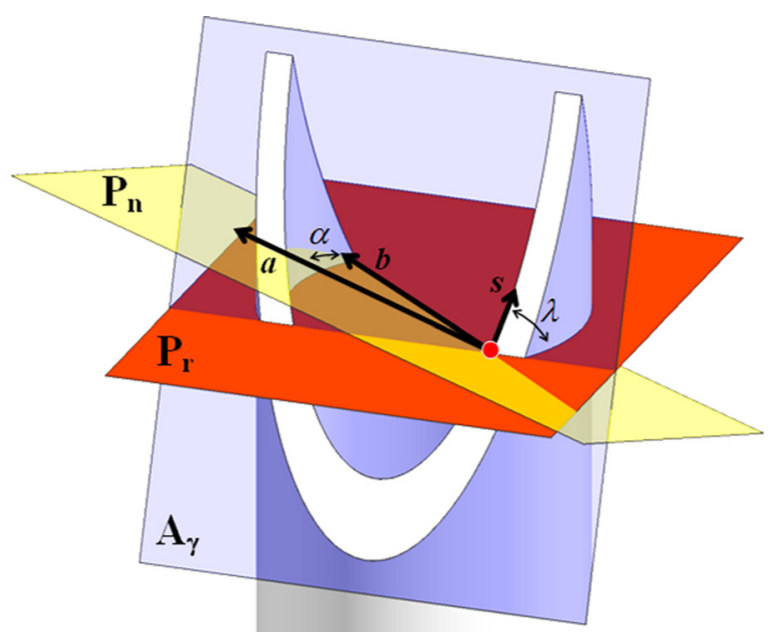

FIG. 2. Definitions of inclination angle $(\lambda)$ and rake angle $(\alpha)$ on a two-plane symmetric needle tip.
It is hypothesized that lower insertion force leads to longer biopsy sample lengths. A predictive force model was previously developed for hollow needle insertion, ${ }^{16}$ which is reviewed in Sec. III, and concluded that high $\lambda$ can lower both insertion force as well as force variation and $\alpha$ has little affect on needle tip cutting force. This knowledge is applied in this study to design a new needle tip geometry, called the enhanced cutting edge (ECE), to increase the $\lambda$ along the cutting edge which will lower insertion force. This study correlates the effect of insertion force to biopsy sample lengths.

The concept to increase $\lambda$ on the leading cutting edge of the needle by creating a sharp needle tip point, known as a lancet point, ${ }^{17}$ has been around since the patent by Huber ${ }^{18}$ in 1946 for hypodermic needles. The lancet point contains two extra surface planes that are ground at the one-plane bevel needle tip to increase $\lambda$ on the leading needle tip point to reduce the needle insertion force. The more recent patents by $\operatorname{Ross}^{19}$ and Gravlee ${ }^{20}$ showed biopsy needle tip designs that improved biopsy cutting efficiency. However, none of these inventors ${ }^{18-20}$ studied the specific effects of changing the rake and inclination angle needle tip geometry.

In this study, the ECE and regular two-plane needles are evaluated for end-cut biopsy of tissue under various levels of internal needle pressure. Experiments of needle insertion into bovine liver are performed using regular and ECE 11 gauge two-plane symmetric needles. The levels of internal needle pressure tested are atmospheric, $0 \mathrm{kPa}$, and the vacuum gauge pressures of -33.9 and $-67.7 \mathrm{kPa}$. These insertion studies compare both the initial cutting force and the biopsy sample length to distinguish the performance of the ECE versus regular needle tip designs.

In this study, the rake and inclination angles of two-plane and ECE needle tips are compared. Next, a needle insertion force model is reviewed. Biopsy experiments are conducted into bovine liver, measuring insertion force and biopsy sample length. Lastly, the results are analyzed and discussed.

\section{TWO-PLANE AND ECE NEEDLE TIP GEOMETRY}

The geometry of the needle cutting edge can be characterized by $\lambda$, and $\alpha{ }^{12}$ Moore et al. ${ }^{12}$ has solved the $\lambda$ and $\alpha$ for a variety of flat plane and curved surfaced needle tips. For the two-plane symmetric needle, as shown in Fig. 3 , the $\lambda$ and $\alpha$ are

$$
\begin{aligned}
& \lambda(\xi, \gamma)=\arcsin \frac{|\cot \xi \sin \gamma|}{\sqrt{1+\cot ^{2} \xi \sin ^{2} \gamma}} \\
& \alpha(\xi, \gamma)=\arccos \sqrt{\cos ^{2} \gamma \sin ^{2} \xi+\sin ^{2} \gamma}
\end{aligned}
$$

where $\xi$ is the bevel angle of the needle and $\gamma$ is the radial coordinate to a point $\mathrm{A}$ on the cutting edge, as illustrated on the two-plane symmetric needle in Fig. 3. An $x y z$ coordinate axis is defined for any needle by the $z$ axis coinciding with the needle axis and the $x$ axis passing through the lowest point of the needle tip profile.

A two-plane symmetric needle contains inclination angles that vary, ${ }^{12} 0 \leq \lambda \leq 90^{\circ}-\xi$, and rake angles that vary, 


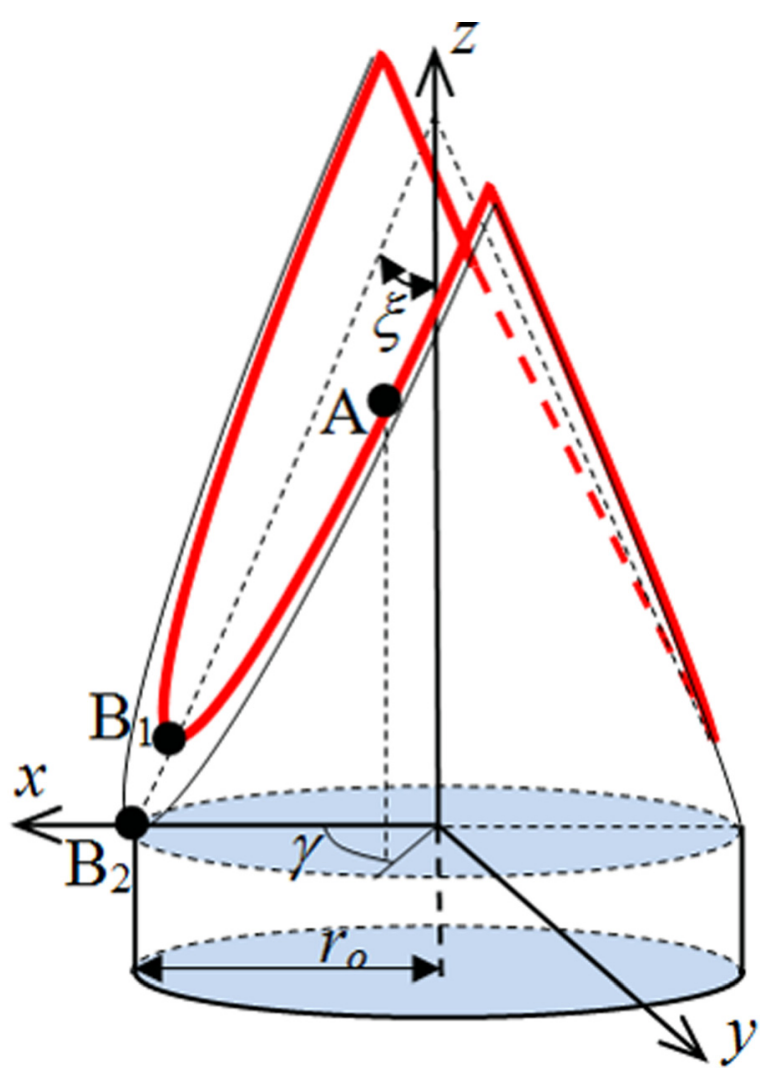

FIG. 3. Regular two-plane symmetric needle defined by the bevel angle $\xi$.
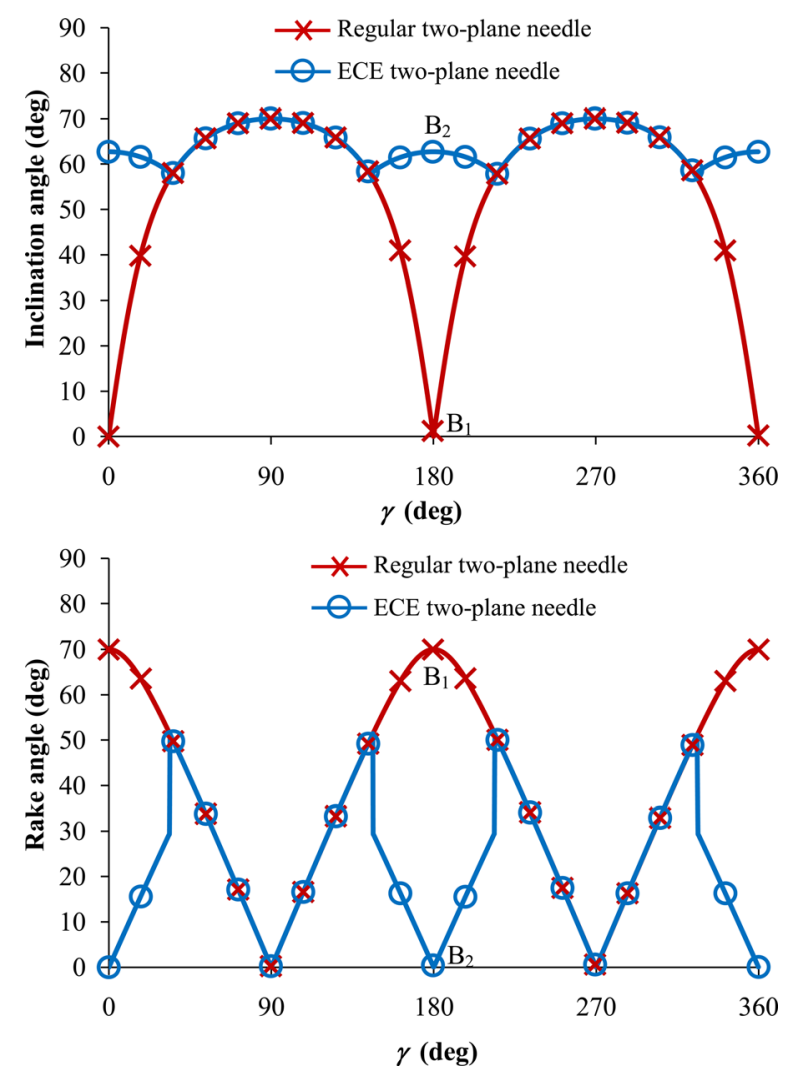

FIG. 4. Inclination $(\lambda)$ and rake angle $(\alpha)$ of regular two-plane symmetric needle and ECE two-plane needle.
$0 \leq \alpha \leq 90^{\circ}-\xi$. Results of $\lambda$ and $\alpha$ for a regular two-plane needle with $\xi=20^{\circ}$ is shown in Fig. 4. The $\lambda=0^{\circ}$ at point $\mathrm{B}_{1}$, known as the heel of the bevel, which is marked in Figs. 3 and 4. Increasing this region's $\lambda$ can improve tissue cutting efficiency and thereby allow for longer biopsy samples to be obtained.

Figure 5(a) shows an ECE two-plane needle that uses a wedge with angle $\varphi$ to cut the angled edges into the heel of the bevel where $\lambda=0^{\circ}$, thereby eliminating this poor cutting condition found at and around $\mathrm{B}_{1}$. The wedge removes material up to point $\mathrm{B}_{2}$, the lowest point in the $z$-direction on the ground surface of the needle tip. The location of $\mathrm{B}_{2}$ is also shown in Figs. 3 and 6(a). The groove in the ECE needle is created using electrical discharge machining (EDM) with a wedge electrode made of graphite as shown in Fig. 5. The cutting edge profile of the needle can be either be continuous as with the example in Fig. 5(a) or discontinuous as with Fig. 5(b).

The $\lambda$ and $\alpha$ for an ECE two-plane needle is found by dividing the needle into sections based on how the needle cutting edge geometry was created. The needle is found to contain two sections shown in Fig. 6. Section 1 is the area where the needle cutting edge is formed by grinding two planes on the needle, Section 2 is the area where the needle cutting edge is formed by EDM cutting. The radial coordinate $\gamma_{e}$, as shown in Fig. 6(c), marks the initial transition from Sec. 2 to Sec. 1. Section 1 occurs when $\gamma_{e} \leq \gamma \leq 180^{\circ}-\gamma_{e}$ and $180^{\circ}+\gamma_{e} \leq \gamma \leq 360^{\circ}-\gamma_{e}$. Section 2 occurs when $0 \leq \gamma \leq \gamma_{e}$, $180^{\circ}-\gamma_{e} \leq \gamma \leq 180^{\circ}+\gamma_{e}$, and $360^{\circ}-\gamma_{e} \leq \gamma \leq 360^{\circ}$.

The $\lambda$ and $\alpha$ for the ECE needle are

$$
\begin{aligned}
& \text { Section 1: } \lambda(\xi, \gamma)=\arcsin \frac{|\cot \xi \sin \gamma|}{\sqrt{1+\cot ^{2} \xi \sin ^{2} \gamma}} \\
& \text { Section 2: } \lambda(\xi, \gamma)=\arcsin \frac{\left|\cot \frac{\phi}{2} \sin \left(\gamma+\frac{\pi}{2}\right)\right|}{\sqrt{1+\cot ^{2} \frac{\phi}{2} \sin ^{2}\left(\gamma+\frac{\pi}{2}\right)}}
\end{aligned}
$$

Section $1: \alpha=\arccos \sqrt{\cos ^{2} \gamma \sin ^{2} \xi+\sin ^{2} \gamma}$

Section 2: $\alpha=\arccos \sqrt{\cos ^{2}\left(\gamma+\frac{\pi}{2}\right) \sin ^{2} \frac{\phi}{2}+\sin ^{2}\left(\gamma+\frac{\pi}{2}\right)}$

Figure 4 illustrates the $\lambda$ and $\alpha$ for an ECE two-plane needle tip with $\xi=20^{\circ}$ and $\varphi=57.5^{\circ}$. The ECE needle contains on average higher $\lambda$ and lower $\alpha$ than the regular two-plane needle. The ECE two-plane needle at point $\mathrm{B}_{2}$ has $\lambda=90^{\circ}-\varphi / 2$ as compared to $\lambda=0^{\circ}$ at point $\mathrm{B}_{1}$ on a regular two-plane needle.

For the continuous cutting edge ECE two-plane needle configuration in Fig. 5(a), the angles of $\varphi, \gamma_{e}$, and $\xi$ are all related where point $\mathrm{E}$ at $\gamma=\gamma_{e}$ is continuous. The distance from $\mathrm{B}_{2}$ to $\mathrm{E}$ in the $z$-direction, is $l_{n}$, as shown in Fig. 6(b) and solved as

$$
l_{n}=(t+q) \cot \xi
$$

where $t$ is the needle wall thickness and $q$ is the distance in the $x$-direction when $y=0$ from the inside needle wall to $\mathrm{E}$ as shown in Figs. 6(b) and 6(c). The length $q$ can be put in terms of $\gamma_{e}$ and $r_{i}$, the inside radius of the needle, as 


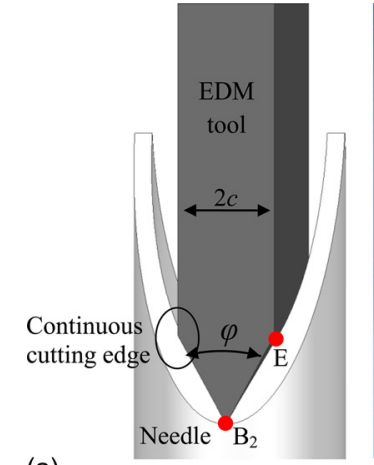

(a)

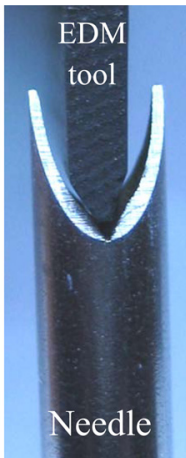

(b)

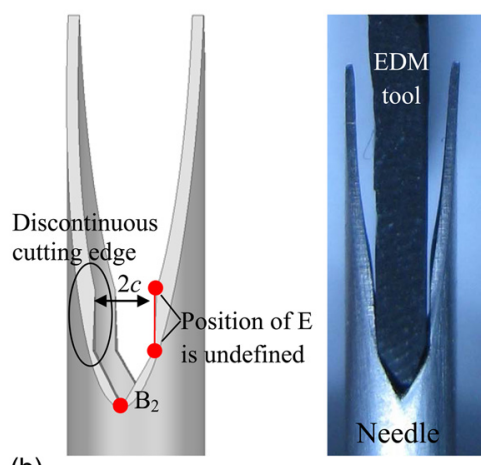

FIG. 5. (a) ECE two-plane needle with a continuous cutting edge and (b) ECE two-plane needle with a discontinuous cutting edge.

$$
q=r_{i}\left(1-\cos \gamma_{e}\right)
$$

The width of the top of the wedge is equal to $2 c$, as shown in Figs. 5(a), 6(a), and 6(c), and $c$ is

$$
c=l_{n} \tan (\varphi / 2)=r_{i} \sin \gamma_{e}
$$

Combining Eqs. (5)-(7) gives the relationship among $\varphi, \gamma_{e}$, and $\xi$

$$
\cot \xi \tan (\varphi / 2)\left(t+r_{i}-r_{i} \cos \gamma_{e}\right)=r_{i} \sin \gamma_{e}
$$

Using trigonometric identities and the quadratic equation, this equation is solved in terms of $\gamma_{e}$ as

$$
\gamma_{\mathrm{e}}=2 \tan ^{-1}\left(\frac{1-\sqrt{1-\tan ^{2}\left(\frac{\varphi}{2}\right) \cot ^{2}(\xi)\left(\frac{t^{2}+2 t r_{i}}{r_{i}^{2}}\right)}}{\tan \left(\frac{\varphi}{2}\right) \cot (\xi) \frac{t+2 r_{i}}{r_{i}}}\right)
$$

If Eq. (9) produces an imaginary solution to $\gamma_{e}$, then the cutting edge must be discontinuous. In the case that the cutting edge of Section 1 is tangent to the cutting edge of Section 2, the square root in Eq. (9) will equal 0 with the given parameters of $t, r_{i}, \varphi$, and $\xi$.

The needle cutting edge is discontinuous when point $\mathrm{E}$ is discontinuous at $\gamma=\gamma_{e}$, as in the example in Fig. 5(b). In this situation, the values of $\varphi, \xi$, and $\gamma_{e}$ are independent of each other and Eq. (9) does not apply. The geometry of a discontinuous ECE two-plane needle is defined by $\varphi, \xi$, and $c$.
While the geometry of a continuous ECE two-plane needle is defined by only $\varphi$ and $\xi$.

\section{NEEDLE INSERTION FORCE MODEL AND $S$ FACTOR}

Moore et al. ${ }^{16}$ developed a predictive force model based on $\lambda$ and $\alpha$ of a needle's cutting edge to predict the force necessary for a hollow needle to initially cut tissue, $F_{N}$. The model was developed by inserting 16 blades of varying $\lambda$ and $\alpha$ into bovine liver and measuring the specific force, thereby allowing for the development of a model $f(\lambda, \alpha)$, as shown in Eq. (10), for specific force needed to cut tissue for a cutting edge of a given $\lambda$ and $\alpha$. Higher $\lambda$ lowers insertion force while $\alpha$ has little affect on insertion force

$$
\begin{aligned}
f(\lambda, \alpha)= & -0.042+0.296 \lambda+0.298 \alpha-0.255 \lambda^{2} \\
& -0.408 \lambda \alpha-0.011 \alpha^{2}+0.083 \lambda^{3} \\
& +0.118 \lambda^{2} \alpha+0.080 \lambda \alpha^{2}-0.059 \alpha^{3}
\end{aligned}
$$

For a two-plane needle at the moment of initial tissue cutting, a portion of the needle is exposed to tissue cutting as shown in Fig. 7. The tissue contacts the needle on the leading edge of the needle tip and on the inside cutting edge, as marked in Fig. 7(a), over a specific range marked as $\theta$ in Fig. 7(b). Moore et al. ${ }^{16}$ experimentally found that this angle $\theta$ changed based on $\xi$ as

$$
\begin{array}{ll}
\theta=9.2 \xi & \xi<19.6^{\circ} \\
\theta=180^{\circ} & \xi>19.6^{\circ}
\end{array}
$$

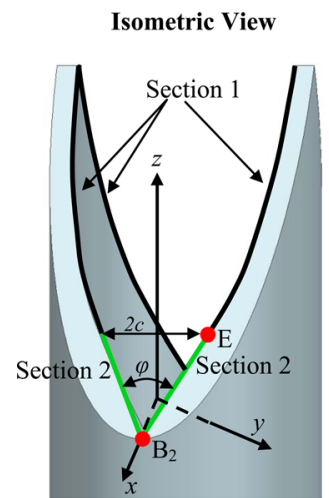

(a)

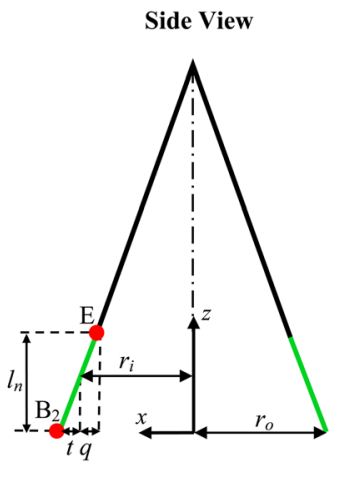

(b)

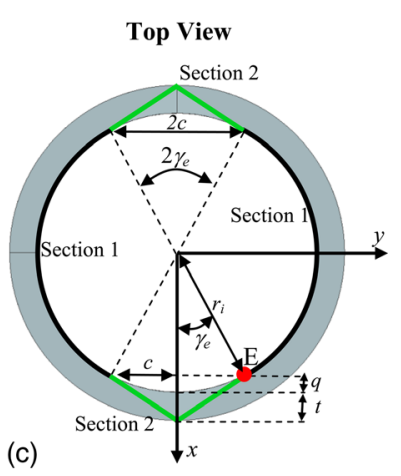

FIG. 6. ECE two-plane needle in (a) isometric view (b) side view, and (c) top view. 


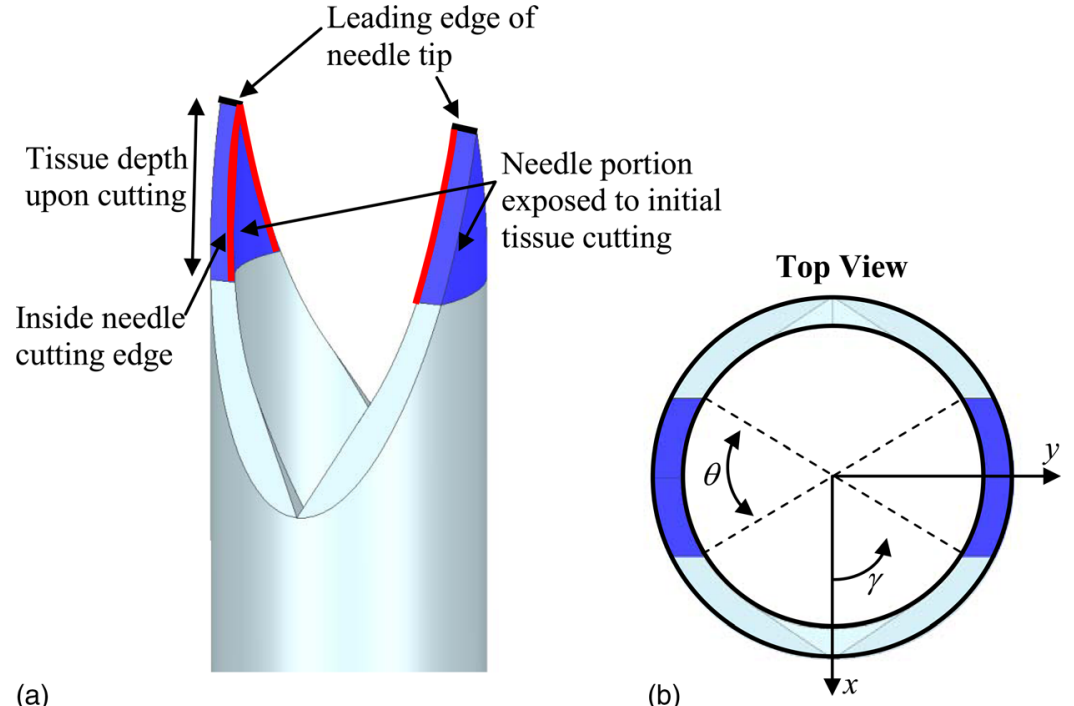

FIG. 7. (a) Side view and (b) top view of needle tip exposed to initial tissue cutting.
Moore et $a l .{ }^{16}$ developed the force model shown in Eq. (12) for a two-plane symmetric needle by integrating $f(\lambda, \alpha)$ around the inside needle tip cutting edge and calculating the cutting force on the leading edge by knowing the leading edge geometry for a two-plane needle is $\lambda=0^{\circ}$ and $\alpha=\frac{\pi}{2}-\xi$.

$$
F_{N}=S \int_{\frac{\pi-\theta}{2}}^{\frac{\pi+\theta}{2}} 2 f(\lambda, \alpha) r_{i} d \gamma+2 t f\left(0, \frac{\pi}{2}-\xi\right)
$$

The variable $S$ is the scale factor used to convert the straight blade cutting to round needle insertion force using the $f(\lambda, \alpha)$ obtained in blade cutting. A round needle distributes force over a larger area of tissue than does the flat blade, thereby making the needle less efficient at cutting tissue than the flat blade when no internal vacuum is applied. The $S$ factor properly scales the needle cutting force to the blade cutting force. Vacuum experiments, described in the Sec. IV, show

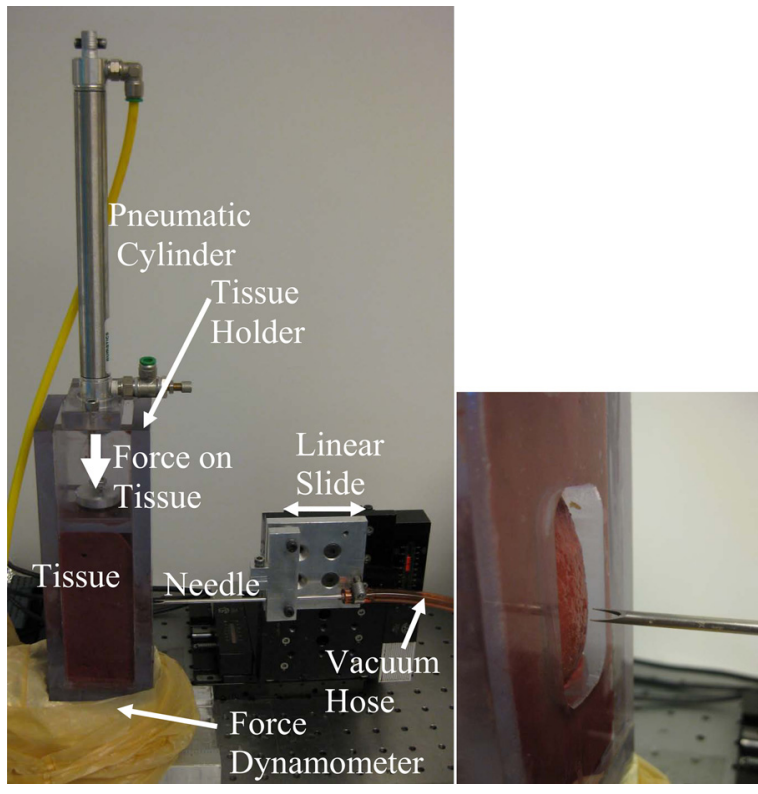

FIG. 8. Experimental setup for needle insertion into bovine liver. the $S$ factor is lower, indicating higher cutting efficiency, for higher levels of vacuum applied.

\section{EXPERIMENTATION}

\section{IV.A. Setup for Needle insertion experiments}

The overview and close-up view of the experimental setup to perform the needle insertion experiments is shown in Fig. 8. The experimental setup uses a Siskiyou Instrument (Grants Pass, Oregon) 200cri linear stage, a Kistler (Winterthur, Switzerland) 9256A1 piezoelectric force dynamometer, and a tissue holder that, via a pneumatic cylinder, applies a static pressure of $15.5 \mathrm{kPa}$ on the top of the bovine liver tissue block to ensure consistent tissue conditions.

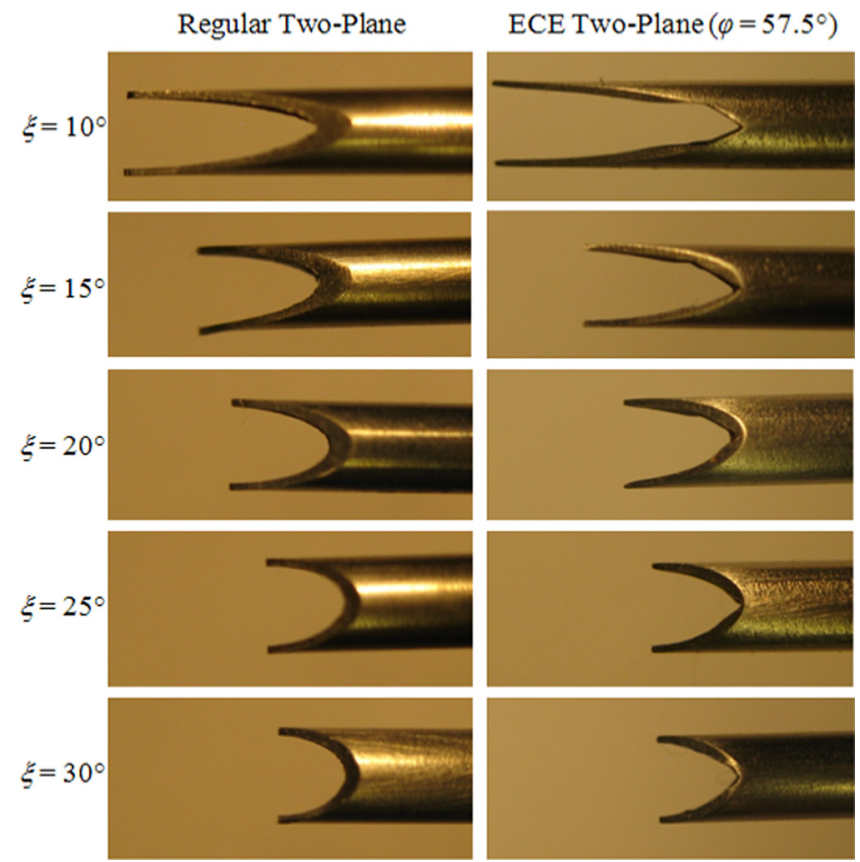

FIG. 9. Regular two-plane symmetric and ECE needles used for experiments. 


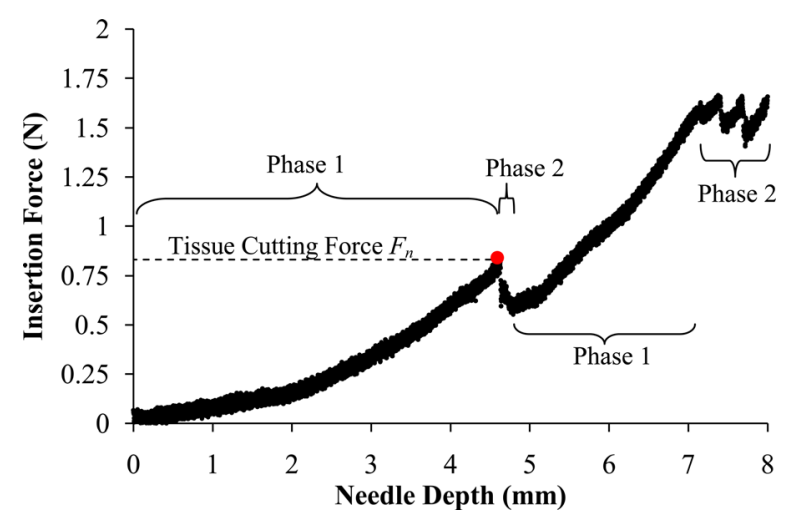

FIG. 10. Needle insertion into bovine liver force example of ECE needle with $\xi=20^{\circ}$.

Bovine liver is a very soft work-material; therefore, consistent tissue holding conditions are critical to produce repeatable and reliable results as demonstrated by Shih et al. ${ }^{21}$ in a study of machining elastomers, another soft workmaterial. The needles were inserted at a rate of $1.5 \mathrm{~mm} / \mathrm{s}$ through $50 \mathrm{~mm}$ length of tissue. A vacuum pump is used to supply a constant vacuum pressure to the inside of the needle for the experiments using the vacuum gauge pressures of -33.9 and $-67.7 \mathrm{kPa}$. After each needle insertion, the sample was then extracted using a solid rod and biopsy length was measured.

\section{IV.B. Needles}

A total of ten needles were manufactured as shown in Fig. 9; five regular two-plane needles and five ECE two-plane needles both having bevel angles, $\xi$, of $10^{\circ}, 15^{\circ}$, $20^{\circ}, 25^{\circ}$, and $30^{\circ}$. The needles are 11 gauge thin wall 316 stainless steel needles (OD $3.05 \mathrm{~mm}$ and ID $2.54 \mathrm{~mm}$ ). All the ECE two-plane needles are machined with the angle $\varphi=57.5^{\circ}$. This angle is used because for $\xi=20^{\circ}$, the wedge cutting edge will be tangent to the inside cutting edge for the 11 gauge thin wall needles. The ECE needles of $\xi=15^{\circ}$ and $\xi=10^{\circ}$ are discontinuous as shown previously in Fig. 5(b). The $c$ value for needles $\xi=10^{\circ}$ and $15^{\circ}$ is $0.69 \mathrm{~mm}$ because the graphite EDM milling tool used to make the needles has a width, $2 c$, of $1.38 \mathrm{~mm}$.

\section{IV.C. Determination of initial tissue cutting force from the experiments}

The force model previously described by Moore et al. ${ }^{16}$ examines the force when the tissue is initially cut $\left(F_{N}\right)$. The force during needle insertion into tissue has been described by Heverly et $a l^{22}$ to occur in two distinct phases, Phase 1 and Phase 2. Phase 1 is the initial phase where the tissue deflects and force increases but no physical tissue cutting occurs. Phase 2 is the second phase where tissue is cut and the force suddenly drops or levels off. If uncut tissue is in front of the needle after Phase 2, then Phase 1 will repeat and the process will start over again. This cycle will continue until the needle insertion is stopped or all the tissue is cut. The first transition between Phase 1 and Phase 2 marks the force $F_{N}$, as illustrated by the example force graph in Fig. 10 which shows the force results for one of the experiments on an ECE needle of $\xi=20^{\circ}$. The experimental force data from all the experiments are examined in this way to determine $F_{N}$.

\section{IV.D. Summary of experimental procedure}

In summary, this study uses five regular two-plane needles and five ECE two-plane needles (both having $\xi=10^{\circ}$, $15^{\circ}, 20^{\circ}, 25^{\circ}$, and $30^{\circ}$ ). The ECE and regular needles are tested at the pressures of $0,-33.9$, and $-67.7 \mathrm{kPa}$. Ten needle insertions are performed for each needle style and pressure level upon which both insertion force and biopsy length are measured for a total of 300 needle insertion experiments.

\section{RESULTS AND ANALYSIS}

Biopsy length and force results along with limiting biopsy performance factors are discussed in Sec. V A, V B, V C, and V D.

\section{V.A. Biopsy length}

\section{V.A.1. Needle geometry effect}

The ECE needle produces longer biopsy results than the regular needle. As illustrated in Fig. 11, the biopsy sample length is on average $22 \%, 30 \%$, and $49 \%$ longer for ECE needles compared to that of regular needles for the internal pressures of
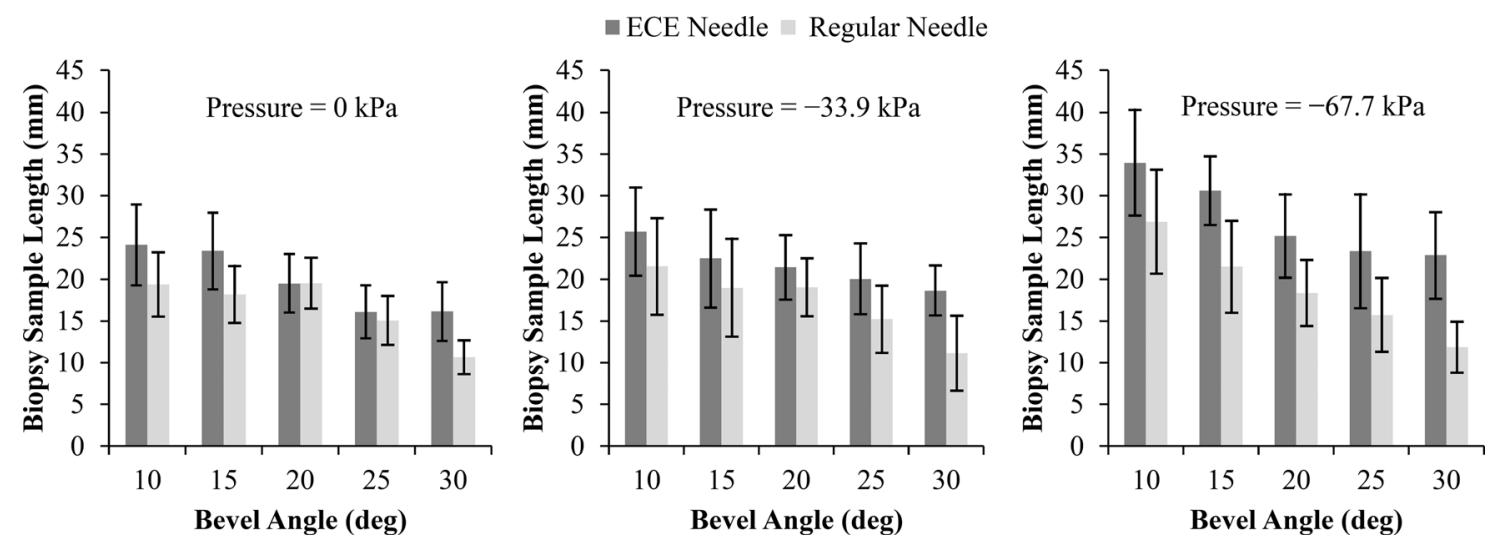

FIG. 11. Biopsy sample length comparing ECE two-plane needles to regular two-plane needles. 

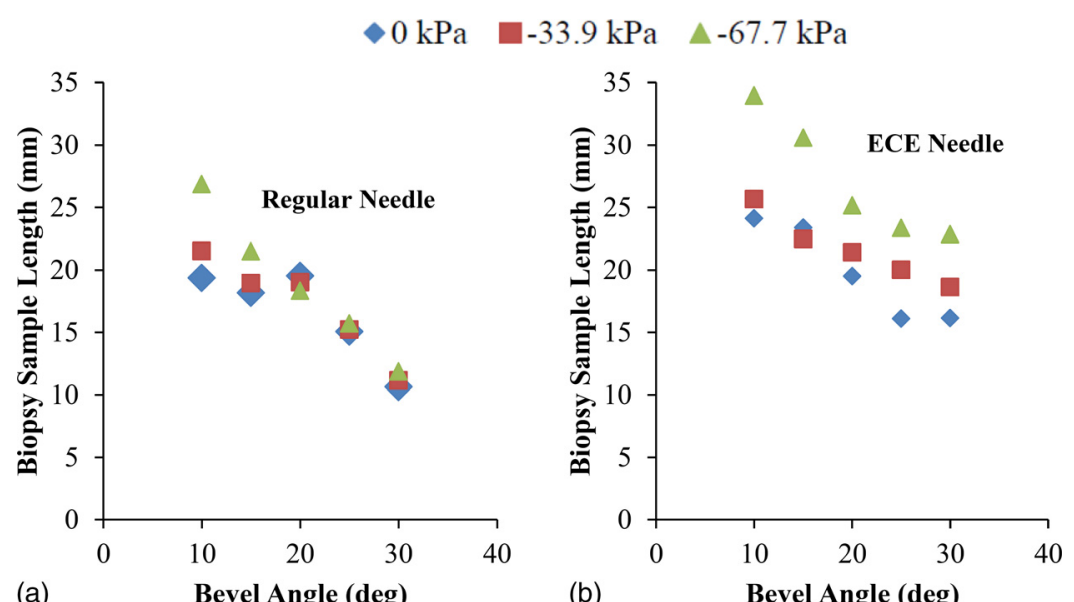

FIG. 12. Biopsy sample length comparing pressure effect for (a) regular and (b) ECE two-plane needles.

$0,-33.9$, and $-67.7 \mathrm{kPa}$, respectively. This demonstrates that the ECE concept of modifying the needle to increase inclination angle on cutting edge is beneficial for end-cut biopsy. The higher inclination angles allow for more efficient needle tip cutting of tissue and, therefore, longer biopsy samples.

Lower bevel angles result in longer biopsy lengths for both ECE and regular two-plane needles. The lower bevel angles correspond to higher inclination angles that are demonstrated to more efficiently cut the tissue. This observation reflects the force model prediction that higher inclination angles are able to cut tissue with less force.

The effect of vacuum is not easily distinguished in Fig. 11; therefore, Fig. 12 is created by rearranging the biopsy length data to compare the three vacuum levels.

\section{V.A.2. Vacuum effect}

For regular two-plane needles shown in Fig. 12(a), the use of vacuum improves biopsy length for $\xi=10^{\circ}$ and $15^{\circ}$ but shows no effect for $\xi=20^{\circ}, 25^{\circ}$, and $30^{\circ}$. Previously Moore et al. ${ }^{16}$ showed that higher $\lambda$ cuts tissue with lower insertion force making the cutting edge more efficient at cutting tissue. The low inclination angles in the regular needles of $\xi=20^{\circ}$, $25^{\circ}$, and $30^{\circ}$ make them inefficient at cutting tissue. The vacuum is only beneficial to biopsy length if the needle tip can efficiently cut the tissue. If the needle tip can efficiently cut tissue, then the tissue sample that enters the needle can be pulled into the needle by the vacuum force which acts to overcome the internal wall needle friction as shown in Fig. 13. If the needle is unable to effectively cut tissue, then the tissue sample does not effectively enter the needle preventing the vacuum force from benefiting the biopsy yield.

For ECE needles, the vacuum level of $-67.7 \mathrm{kPa}$ produces on average biopsy lengths that are $41,31,29,45$, and $42 \%$

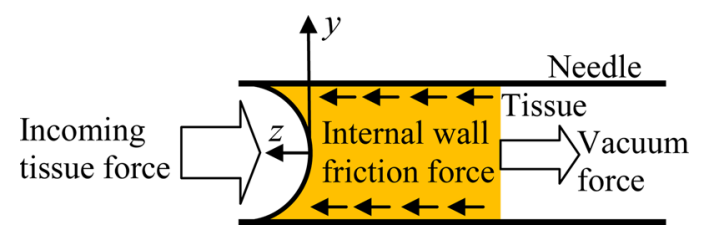

FIG. 13. Internal wall friction force repels the motion of the incoming tissue while the vacuum force helps to pull the sample into the needle. longer compared to no vacuum for $\xi=10^{\circ}, 15^{\circ}, 20^{\circ}, 25^{\circ}$, and $30^{\circ}$, respectively, as illustrated in Fig. 12(b). The ECE needle tip's high inclination angle makes it effective at cutting the tissue and the vacuum allows the needle to overcome the internal tissue friction shown in Fig. 13; thereby, leading to longer biopsy samples for vacuum assisted ECE needles.

\section{V.B. Biopsy force}

\section{V.B.1. Needle geometry effect}

The ECE needle produces lower needle insertion forces than regular needles for $\xi=20^{\circ}, 25^{\circ}$, and $30^{\circ}$ as shown in Fig. 14. The initial insertion force is on average $20 \%, 9 \%$, and $5 \%$ higher for regular compared to ECE two-plane needles for the internal needle pressures of $0,-33.9$, and $-67.7 \mathrm{kPa}$, respectively, on the needles of $\xi=20^{\circ}, 25^{\circ}$, and $30^{\circ}$. Cutting force differences between ECE and regular needles are less noticeable at higher vacuum levels because increased vacuum lowers cutting force on all needles, thereby making differences in performances less pronounced.

The needles of $\xi=10^{\circ}$ and $\xi=15^{\circ}$ show little change because the ECE needle geometry differs from the regular two-plane needle only at the far back of the needle tip and the initial tissue cutting occurs at the front of the needle tip when $\xi=10^{\circ}$ and $15^{\circ}$. This lower force for higher inclination angles corresponds to previous findings by Moore et al. ${ }^{16}$

For both ECE and regular two-plane needles, lower bevel angles result in lower biopsy insertion forces. Again demonstrating that less force is required to cut tissue when using needles containing higher inclination angles.

\section{V.B.2. Vacuum effect}

Vacuum lowers the average insertion force for both regular and ECE two-plane needles of $\xi=15^{\circ}, 20^{\circ}, 25^{\circ}$, and $30^{\circ}$ as illustrated in Figs. 15(a) and 15(b), respectively. When vacuum is used the vacuum force helps to apply the force necessary to cut the tissue which leads to lower insertion forces. The vacuum also aids in overcoming the internal needle friction as discussed in Sec. V A 2.

For the needles of $\xi=10^{\circ}$, the vacuum level appears to have little effect on the insertion force. This happens because the initial tissue cutting occurs at the very front of the needle 

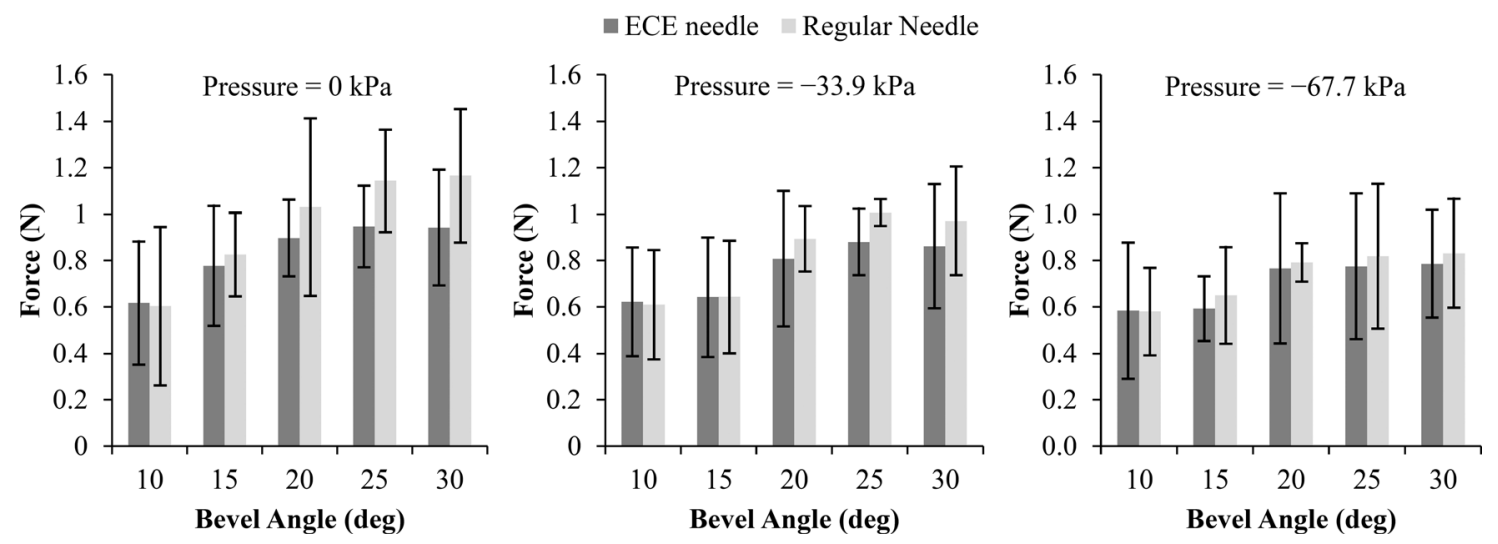

FIG. 14. Needle insertion force comparing ECE two-plane needles to regular two-plane needles.

$\xi=10^{\circ}$. In this configuration, the vacuum is unable to apply a vacuum force on the tissue because mostly air is being pulled into the needle.

\section{V.C.3. S-factor variation for vacuum effect}

The $S$ factor decreases at higher vacuum levels for both ECE and regular needles showing that vacuum improves cutting efficiency. The $S$ factor in Eq. (12) is determined using a least squares fit to the force data for the regular two-plane needles and ECE needles, for all the three tested levels of internal needle pressure as shown in Fig. 16. Figure 17 shows that the $S$ factor for both types of needles decreases at lower pressures. This indicates higher cutting efficiency is obtained when lower vacuum pressure is applied to the inside of the needles.

\section{V.C. Comparing biopsy length to biopsy force}

Needles of higher inclination angle that produce lower insertion forces also produce on average longer biopsy sample lengths. Figures 18(a)-18(c) shows the relationship between needle insertion forces and biopsy length for internal needle pressures of $0,-33.9$, and $-67.7 \mathrm{kPa}$, respectively. Least squares fit linear lines are illustrated for both ECE and regular needles. It is shown that for both ECE and regular needles lower needle insertion forces coincide with longer biopsy lengths as shown with least squares fit linear lines all having negative slopes. Lower insertion forces are an indication of more efficient tissue cutting. More efficient tissue cutting leads to longer biopsy sample lengths.

Figure 18 also shows the $R^{2}$ values for the linear least squares best fit lines. Higher levels of vacuum lead to higher $R^{2}$ values which indicate a more linear relationship. This improved linearity is a direct result of the vacuum force overcoming the internal needle friction which hinders longer biopsy lengths from being obtained. Figure 18(a) shows that for the internal needle pressure of $0 \mathrm{kPa}$ both ECE and regular needles lower force corresponds to little improvement in biopsy length when $\xi=10^{\circ}$ and $20^{\circ}$, the needles that produce the two lowest needle insertion forces. However, for the internal needle pressure of $-33.9 \mathrm{kPa}$ [Fig. 18(b)] and $-67.7 \mathrm{kPa}$ [Fig. 18(c)], lower forces correspond to significantly longer biopsy lengths even for the highest biopsy lengths found when $\xi=10^{\circ}$ and $20^{\circ}$.

Higher vacuum allows for a steeper slope in force to biopsy length results as shown in Fig. 18. This is a result of vacuum creating both lower needle insertion forces and longer biopsy lengths. The slope of both ECE and regular needles are very similar for the vacuum pressures of $-33.9 \mathrm{kPa}$ and $-67.7 \mathrm{kPa}$. This occurs because internal needle friction

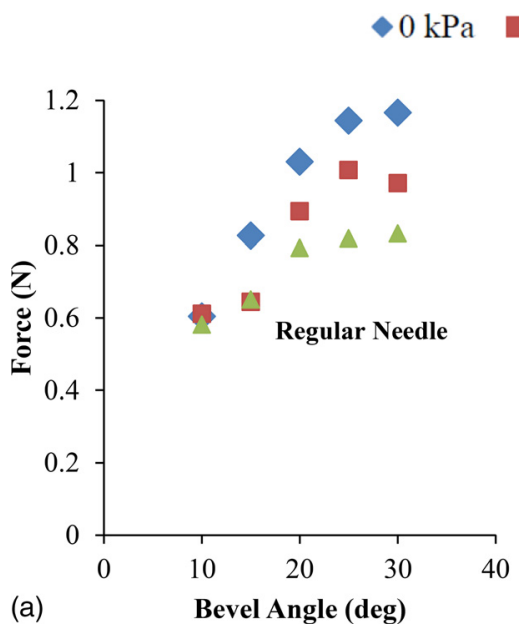

$-33.9 \mathrm{kPa} \triangle-67.7 \mathrm{kPa}$

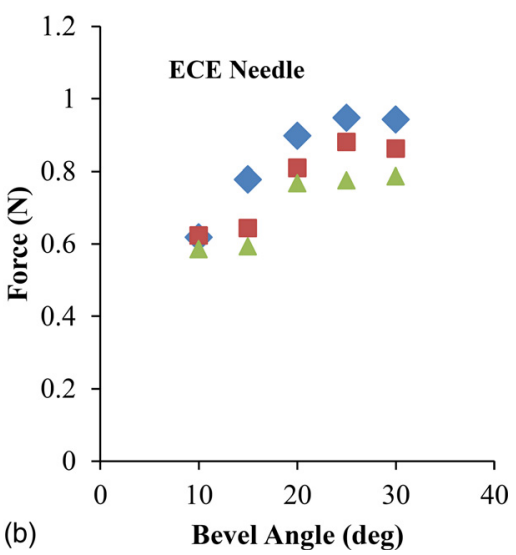

FIG. 15. Needle insertion force comparing pressure effect for (a) regular and (b) ECE two-plane needles. 


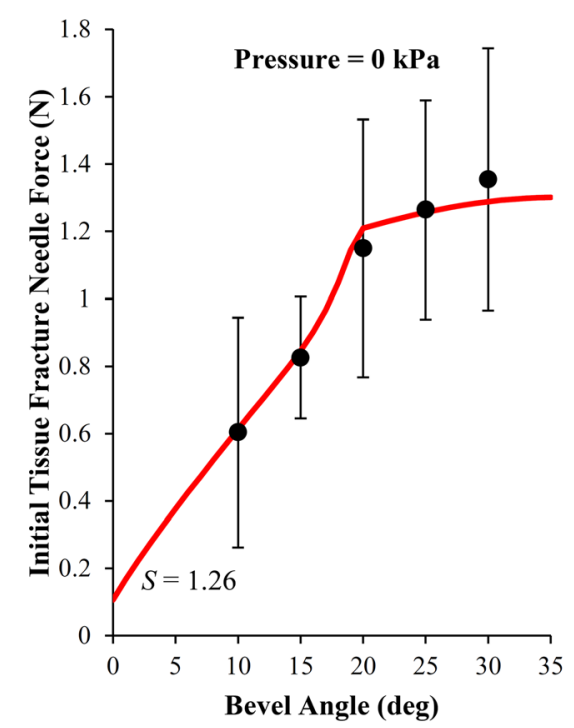

\section{Regular Two-Plane Needles}
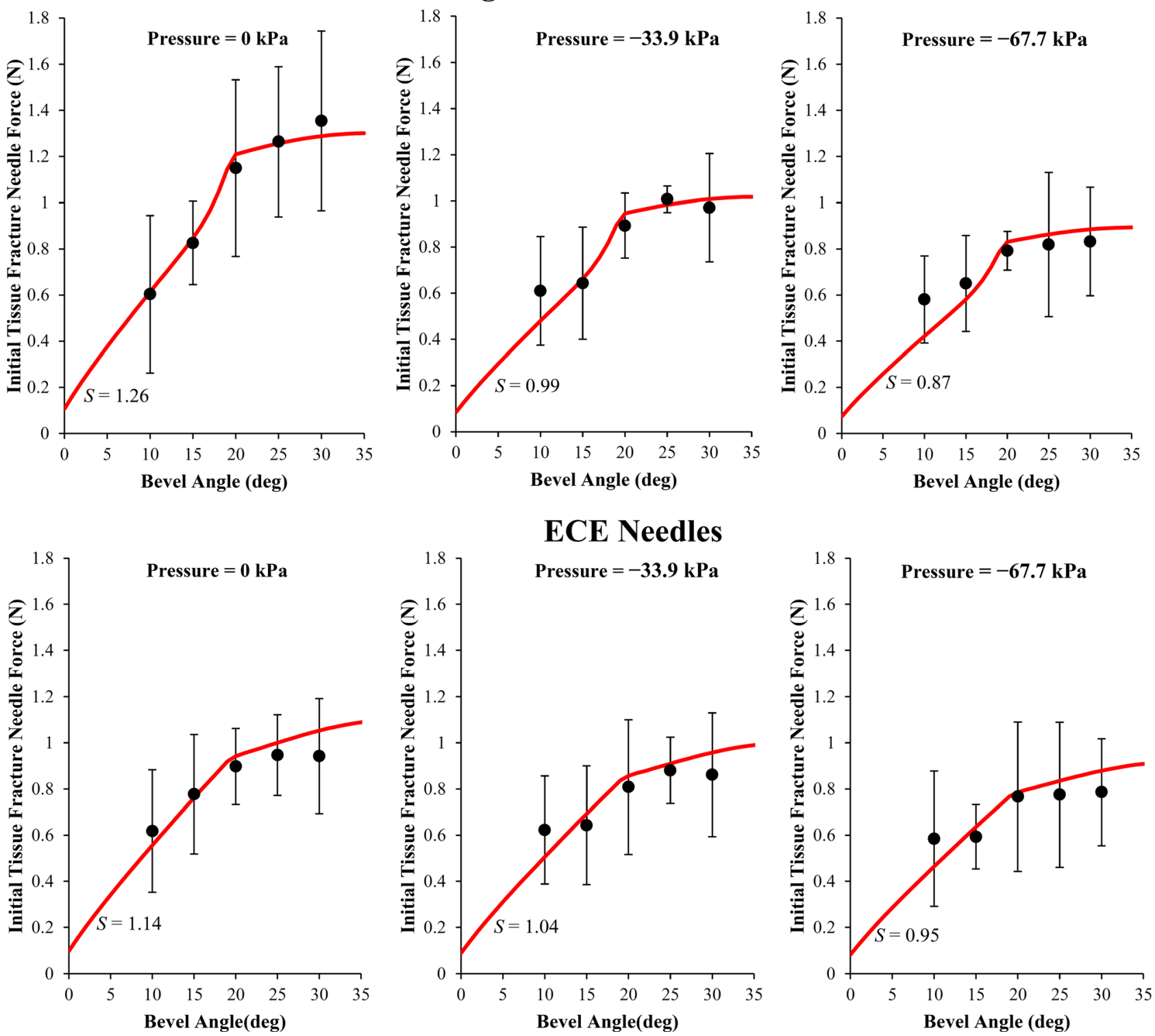

FIG. 16. Force model prediction with given $S$ factors for regular two-plane and ECE needles.

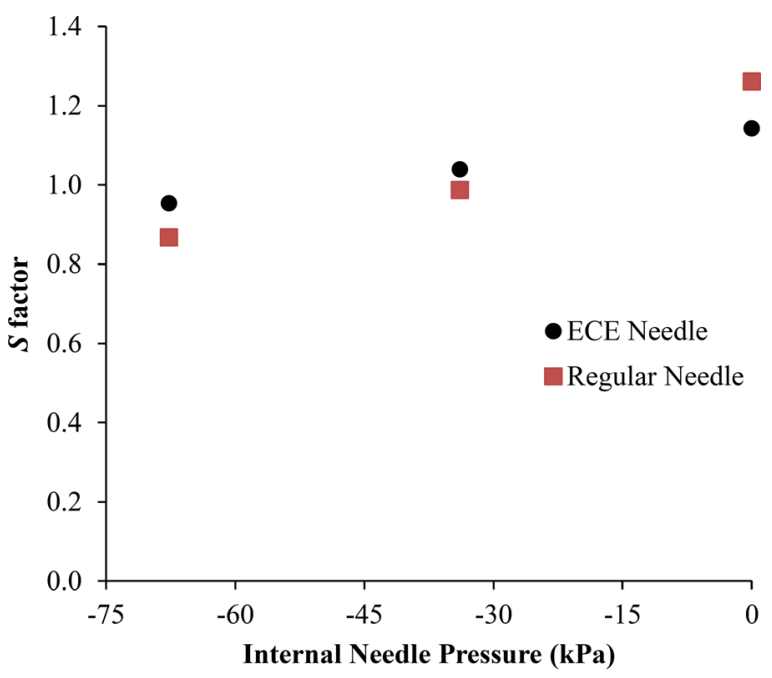

FIG. 17. The effect of needle vacuum pressure on $S$ factor. is not hindering the biopsy length acquisition; therefore, the force of initial insertion is directly related to biopsy length.

\section{V.D. Limiting biopsy performance factors}

The performance of the ECE needle is limited by the needle tip length. Results showed that lower values of $\xi$ improved biopsy performance but the needle tip length, which equals $r_{i} / \tan \xi$, increases greatly at lower $\xi$ values. Too long of a needle tip length will cause the needle tip to become weaker which puts the needle at risk for bending or breaking inside the patient.

Results showed that higher vacuum can allow for longer biopsy lengths. However, too high of a vacuum may cause the tissue sample to be pulled into the vacuum hose making it difficult to be retrieved. High vacuum levels may create fragmented biopsy samples which cause less accurate diagnosis. $^{2}$ 

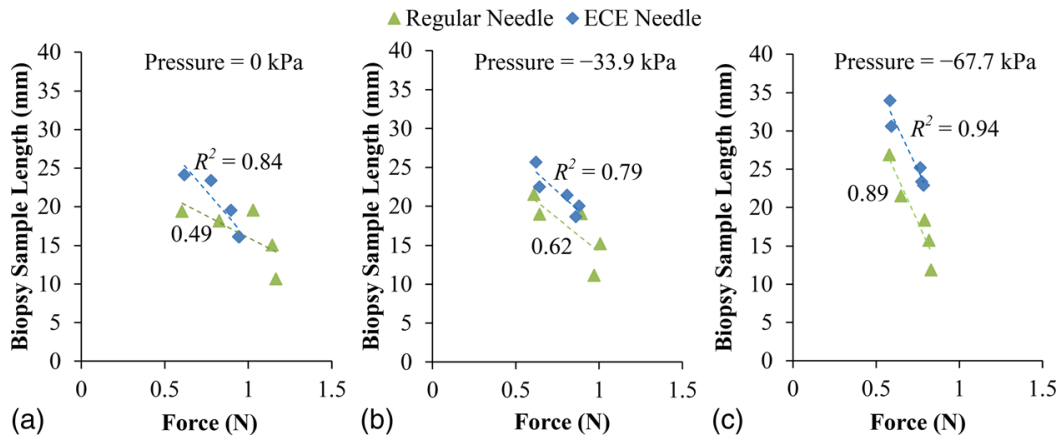

FIG. 18. Needle insertion force compared to biopsy length with least squares best fit lines for needle pressures of (a) $0 \mathrm{kPa}$, (b) $-33.9 \mathrm{kPa}$, and (c) $-67.7 \mathrm{kPa}$.

\section{CONCLUSIONS}

This study demonstrated that needles of higher inclination angle that produce lower insertion forces also produce on average longer biopsy sample lengths. The novel ECE needle tip design was proposed and experimentally determined to outperform the regular two-plane symmetric needle by on average yielding longer biopsy samples. Biopsy sample length is on average 22\%, 30\%, and 49\% longer for ECE needle compared to that of regular needle for the internal pressures of $0,-33.9$, and $-67.7 \mathrm{kPa}$, respectively. The use of vacuum further improved the ECE needle tips biopsy sample length. For ECE needles the vacuum level of -67.7 $\mathrm{kPa}$ produces on average biopsy lengths that are 41, 31, 29, 45 , and $42 \%$ longer compared to no vacuum for $\xi=10^{\circ}$, $15^{\circ}, 20^{\circ}, 25^{\circ}$, and $30^{\circ}$, respectively.

The force results showed the ECE needle could be inserted with less initial insertion force than the two-plane needle for needles where the needle tip was fully contacting the tissue upon insertion. Vacuum was also showed to help lower insertion forces. The $S$ factor of the needle force model was shown to decrease upon increasing the vacuum, indicating a more efficient tissue cutting can take place with the use of vacuum. Future work will focus on optimizing an ECE needle and vacuum level that is safe for patient use and does not lead to damaged or difficult to retrieve biopsy samples.

\section{ACKNOWLEDGMENTS}

This research work is sponsored by the National Science Foundation (NSF) Award CMMI\#0825795, National Natural Science Foundation of China (Award No. 50775119), and supported by the University of Michigan Radiation Oncology Department.

\footnotetext{
a) Author to whom correspondence should be addressed. Electronic mail: jzm14@psu.edu

${ }^{1}$ K. Fink, G. Hutarew, A. Pytel, and N. Schmeller, "Prostate biopsy outcome using 29 mm cutting length," Urol. Int., 75(3), 209-212 (2005).

${ }^{2}$ K. Iczkowski, G. Casella, R. Seppala, G. Jones, B. Mishler, J. Qian, and D. Bostwick, "Needle core length in sextant biopsy influences prostate cancer detection rate," Urology, 59(5), 698-703 (2002).

${ }^{3}$ G. Ubhayakar, W. Li, C. Corbishley, and U. Patel, "Improving glandular coverage during prostate biopsy using a long-core needle: Technical performance of an end-cutting needle," BJU Int. 89(1), 40-43 (2002).

${ }^{4}$ C. D. Lehman and T. Aikawa, "MR-guided vacuum-assisted breast biopsy: Accuracy of targeting and success in sampling in a phantom model," Radiology 232(3), 911-914 (2004).
}

${ }^{5}$ T. K. Podder, D. P. Clark, J. Sherman, D. Fuller, E. Messing, D. Rubens, J. Strang, R. Brasacchio, L. Liao, W. S. Ng, and Y. Yu, "in vivo motion and force measurement of surgical needle intervention during prostate brachytherapy," Med. Phys. 33, 2915-2922 (2006).

${ }^{6}$ B. Maurin, L. Barbe, B. Bayle, P. Zanne, J. Gangloff, M. de Mathelin, A. Gangi, L. Soler, and A. Forgione, "In vivo study of forces during needle insertions," edited by T. M. Buzug and T. C. Lueth, Perspectives in image-guided surgery (World Scientific Pub Co, Singapore, 2004), pp. 415-422.

${ }^{7}$ T. K. Podder, D. P. Clark, J. Sherman, D. Fuller, E. Messing, D. J. Rubens, J. G. Strang, Y. D. Zhang, W. O'Dell, W. S. Ng, and Y. Yu, "Effects of tip geometry of surgical needles: an assessment of force and deflection," 3rd European Medical and Biological Engineering Conference, (Prague, Czech Republic, 2005).

${ }^{8}$ M. O'Leary, C. Simone, T. Wahio, K. Yoshinaka, and A. Okamura, "Robotic needle insertion: effects of friction and needle geometry," in the 2003 IEEE International Conference on Robotics and Automation (Taipei, Taiwan, 2003), pp. 1774-1780.

${ }^{9}$ H. Kataoka, T. Washio, M. Audette, and K. Mizuhara, "A model for relations between needle deflection, force, and thickness on needle penetration," in the Proceedings of the 4th International Conference on Medical Image Computing and Computer-Assisted Intervention (Utrecht, The Netherlands, 2001), pp. 966-974.

${ }^{10}$ A. M. Okamura, C. Simone, and M. O'Leary, "Force modeling for needle insertion into soft tissue," IEEE Trans. Biomed. Eng. 51, 1707-1716 (2004).

${ }^{11}$ R. Webster, J. Kim, N. Cowan, G. Chirikjian, and A. Okamura, "Nonholonomic modeling of needle steering," Int. J. Robot. Res. 25(5-6), 509-525 (2006).

${ }^{12}$ J. Moore, Q. Zhang, C. McGill, P. McLaughlin, H. Zheng, and A. Shih, "Cutting edge rake and inclination angles modeling for plane needles," J. Manuf. Sci. Eng. 132(5), 051005 (2010).

${ }^{13}$ J. Moore, Q. Zhang, C. McGill, H. Zheng, P. McLaughlin, and A. Shih, "Modeling cutting edge geometry for plane and curved needle tips," Proc. Inst. Mech. Eng., Part B (accepted).

${ }^{14}$ J. Moore, C. McGill, Q. Zhang, P. McLaughlin, H. Zheng, and A. Shih, "Blade oblique cutting of tissue for investigation of biopsy needle insertion," Trans. NAMRI/SME 37, 49-56 (2009).

${ }^{15} \mathrm{~J}$. Moore and A. Shih, "Tissue oblique cutting flow angle and needle insertion contact length,” Trans. NAMRI/SME 38, 711-718 (2010).

${ }^{16}$ J. Moore, K. Malukhin, A. Shih, and K. Ehmann, "Hollow needle tissue insertion force model," CIRP Ann. - Manuf. Technol. 60, 157-160 (2011).

${ }^{17}$ T. Kucklick, The Medical Device R\&D Handbook (Taylor \& Francis, Boca Raton, 2006).

${ }^{18}$ R. L. Huber, "Hypodermic needle," U.S, patent 2,409,979 (22 October 1946).

${ }^{19}$ Chauncy F. Ross, “Hypodermic needle," U.S. patent 6,702,790 (9 March 2004).

${ }^{20}$ Joseph F. Gravlee, Jr., "Hypodermic needle," U.S. patent 5,733,266 (31 March 1998).

${ }^{21}$ A. Shih, M. Lewis, and J. Strenkowski, "End milling of elastomersFixture design and tool effectiveness for material removal," J. Manuf. Sci. Eng. 126,115-123 (2004).

${ }^{22}$ M. Heverly, P. Dupont, and J. Triedman, "Trajectory optimization for dynamic needle insertion," International Conference on Robotics and Automation (Barcelona, Spain, 2005), pp. 1658-1663. 